\section{Es kommt dicke ...}

Bis zum Jahr 2020 werden in mehreren der OECD-Mitgliedstaaten zwei Drittel der Bewohner übergewichtig sein. Vor 1980 war es nur jeder zehnte.

Jugendliche, die im Alter von 17 Jahren adipös sind, haben ein fast siebenfach erhöhtes Risiko, in den kommenden 25 Jahren an terminaler Niereninsuffizienz zu erkranken.

Im Durchschnitt nimmt jeder Einwohner aus Industrienationen 8-12 g Salz zu sich. Das ist etwa doppelt so viel wie von der WHO empfohlen.

Obesity update 2012, OECD; Arch Intern Med 2012 (online first); Dorner TE et al, Herz 2012

\section{Weniger Diabetes bei Teetrinkern}

In einer Studie mit Teilnehmern aus 50 Nationen war Schwarzteekonsum invers mit der Häufigkeit von Diabetes verknüpft. Die Statistik basiert auf Daten des World Health Survey, einer von der WHO in Auftrag gegebenen jährlichen Gesundheitsbefragung. Bei der Jahresmenge an konsumiertem Schwarztee pro Einwohner lagen die Briten mit 1,81 kg hinter den Iren $(2,16 \mathrm{~kg})$. Deutschland landete mit knapp $0,2 \mathrm{~kg}$ im hinteren Mittelfeld. Das Schlusslicht bildete Südkorea $(0,0007 \mathrm{~kg})$.

Beresniak A et al, BMJ Open 2012, 2:e000648

\section{Prost Mahlzeit}

Zwischen Zimtsternen und Mettensau gewichtige Beiträge zur (Über-)Ernährung serviert zu bekommen, mag nicht jedem schmecken. Denn Abnehmen ist Schwerarbeit, und weihnachtliche Kalorien scheinen mindestens doppelt anzusetzen. Wie man den ungeliebten Speck wieder los wird, erfahren Sie neben anderen Ernährungsthemen in unserem Schwerpunkt. Eine schmackhafte Lektüre wünscht

\section{Dr. Sonja Kempinski}

Chefredakteurin

\section{Mit Geld-Leckerli wird gut abgespeckt}

Prämien wirken doch als Anreiz zum Abnehmen - zumindest bei Frauen. Zu diesem Ergebnis kommt eine Studie des Rheinisch-Westfälischen Instituts für Wirtschaftsforschung e.V. (RWI) mit rund 700 Patienten im Alter zwischen 18 und 75 Jahren. Nach ihrem Aufenthalt in einer Rehabilitationsklinik sollten die Patienten 6-8\% ihres Körpergewichts innerhalb von vier Monaten abnehmen. Die Patienten wurden zufällig drei Gruppen zugeteilt. In der ersten Gruppe sollte niemand Geld erhalten, in der zweiten jeder $150 €$, in der dritten jeder $300 €$, wenn er das Behandlungsziel erreicht oder überschreitet. Nach vier Monaten nahmen die Teilnehmer der Prämiengruppe durchschnittlich 5\% ihres Ausgangsgewichts ab, die der Kontrollgruppe 2,3\%. Eine weitere Erkenntnis: Je höher der versprochene Geldbetrag, desto mehr Pfunde purzeln: Frauen, denen $300 €$ Prämie in Aussicht gestellt worden war, nahmen um 1,4 Prozentpunkte mehr von ihrem Ausgangsgewicht ab als die Teilnehmerinnen der Gruppe mit geringerer Prämie. Bei Männern hatte die Höhe der Prämie keinen zusätzlichen Effekt.

Pressemitteilung des Rheinisch-Westfälischen Instituts für Wirtschaftsforschung vom 25.10.2012

\title{
Soja hilft Frauen-Hirnen nicht
}

Eine sojareiche Diät hat bei postmenopausalen Frauen insgesamt keinen nennenswerten Effekt auf kognitive Fähigkeiten. So das Ergebnis einer Studie der Universität Stanford. Darin erhielten 313 postmenopausale Frauen über 2,5 Jahre täglich 25 g eines isoflavonreichen Sojaprodukts mit den Inhaltsstoffen Genistein, Daidzein und Glyzitein oder Placebo (gleich aussehendes Milchproteinprodukt ohne Isoflavone).

Die Adhärenz war in beiden Gruppen mit 90 und $89 \%$ exzellent. Beide hatten sich in einem zusammengesetzten neuropsychologischen Score aus 14 ver- schiedenen Parametern insgesamt verbessert, ein Unterschied zwischen den Gruppen war jedoch nicht zu erkennen. Die auch als „Phytoöstrogene“ bezeichneten Isoflavone hatten lediglich zu einer Verbesserung im Parameter „visuelles episodisches Gedächtnis“ (zum Beispiel Gesichter erinnern) geführt.

In allen anderen Punkten, etwa beim räumlichen Vorstellungsvermögen, Auswendiglernen von Aufzählungen oder beim logischen Gedächtnis, lag die Placebogruppe gleichauf.

Henderson VW et al, Neurology 2012, 78:1841

\section{Lachs senkt ADHS-Risiko}

Bei Kindern von Frauen, die in der Schwangerschaft mehr als zweimal pro Woche Fisch gegessen hatten, ist das ADHS-Risiko geringer. Zwei auf den ersten Blick widersprüchlich erscheinende Botschaften in puncto Ernährung bei Schwangeren liefert jetzt eine USStudie mit nahezu 400 Kindern: Bei den Kindern von Frauen, die während der Schwangerschaft hohe Quecksilberwerte im Haar aufwiesen, war das ADHSRisiko erhöht, bei den Nachkommen von Frauen, die in der Schwangerschaft mehr als zweimal pro Woche Fisch gegessen hatten, hingegen verringert. Die Mütter der untersuchten Kinder hatten wohl überwiegend Fisch mit geringem Quecksilbergehalt wie Lachs und Schellfisch verzehrt, schließen die Forscher um Dr. Susan Korrick vom Brigham and Women's Hospital in Boston/Massachusetts.

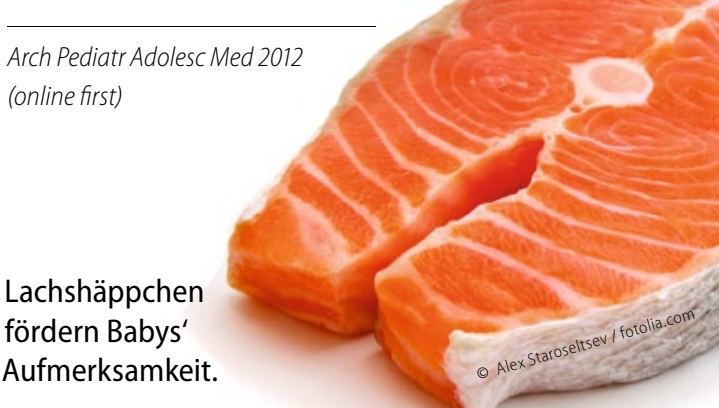
fördern Babys' Aufmerksamkeit. 